\title{
Producción científica del Ecuador en el ámbito agropecuario: caso del Instituto Nacional de Investigaciones Agropecuarias, periodo 2014-2019
}

\author{
William Viera-Arroyo*, Víctor Sánchez-Arizo*, Jorge Merino-Toro*, Juan Domínguez-Andrade** \\ * Dirección de Investigaciones, Instituto Nacional de Investigaciones Agropecuarias (INIAP), Quito (Ecuador). \\ Correo-e: william.viera@iniap.gob.ec | ORCID iD: https://orcid.org/0000-0003-4472-4126 \\ Correo-e: victor.sanchez@iniap.gob.ec | ORCID iD: https://orcid.org/0000-0001-6904-1183 \\ Correo-e: jorge.merino@iniap.gob.ec | ORCID iD: https://orcid.org/0000-0002-5632-2194 \\ ** ESPAE, Graduate School of Management, Escuela Superior Politécnica del Litoral, Guayaquil (Ecuador) \\ Correo-e: jdomingue@espol.edu.ec | ORCID iD: https://orcid.org/0000-0002-7802-2459
}

Recibido: 20-06-20; 2a versión: 17-09-20; Aceptado: 17-09-20.

Cómo citar este artículo/Citation: Viera-Arroyo, W.; Sánchez-Arizo, V.; Merino-Toro, J.; Domínguez-Andrade, J. (2020). Producción científica del Ecuador en el ámbito agropecuario: caso del Instituto Nacional de Investigaciones Agropecuarias, periodo 2014-2019. Revista Española de Documentación Científica, 43 (4), e280. https://doi.org/10.3989/redc.2020.4.1722

\begin{abstract}
Resumen: La producción científica en Ecuador ha seguido un patrón similar a la evolución de otros países latinoamericanos, existiendo escasa generación de documentos indexados; pero en los últimos años se ha incrementado. El INIAP ha plasmado los resultados de sus investigaciones en publicaciones técnicas, dípticos, guías, manuales, entre otros. Desde el año 2014 ha tenido un aumento sostenido en su producción científica, debido a nueva reglamentación de acreditación e incentivos a investigadores. El objetivo de la presente investigación fue realizar un análisis de la producción científica del INIAP durante el periodo 2014-2019. Los resultados mostraron un aumentó 3,1 veces durante el periodo analizado, superando el crecimiento latinoamericano en ese mismo tiempo. Se determinó que más del $54 \%$ de las publicaciones del INIAP tienen colaboración internacional y el 62\% de publicaciones están indexadas en revistas con índice SJR. Estos resultados indican que INIAP ha incrementado su producción y calidad científica en investigación agropecuaria.
\end{abstract}

Palabras clave: producción científica; análisis bibliométrico; investigación agropecuaria; Ecuador.

Scientific production of Ecuador in the agricultural field: case of the National Institute of Agricultural Research, period 2014-2019

Abstract: Scientific production in Ecuador has followed a pattern similar to the evolution of other Latin American countries, with little production of indexed documents but, in recent years, this has increased due to current regulations in the country. INIAP has expressed its research results in technical publications, leaflets, guides and manuals, among others. Since 2014, there has been a sustained increase in its scientific production. The objective of this study is to carry out an analysis of the scientific production of INIAP during the 2014-2019 period. Results showed an increase of 3.1 times during the analyzed period, surpassing the Latin American growth in the same time. It was determined that more than $54 \%$ of INIAP's publications include international collaboration and $62 \%$ of publications are indexed in journals with SJR index. These results indicate that INIAP has increased its scientific production and quality in agricultural research.

Keywords: scientific production; bibliometric analysis; agricultural research; Ecuador.

Copyright: (c) 2020 CSIC. Este es un artículo de acceso abierto distribuido bajo los términos de la licencia de uso y distribución Creative Commons Reconocimiento 4.0 Internacional (CC BY 4.0). 


\section{INTRODUCCIÓN}

La publicación de un artículo científico en una revista especializada constituye el último paso del ciclo de investigación, que permite difundir entre la comunidad científica el resultado del trabajo investigativo (Chaple, 2015; Inglesi-Lotz y Pouris, 2011). En los últimos años se ha incrementado el interés por desarrollar instrumentos que permitan el estudio de varias áreas científicas debido a que ha aumentado el número de publicaciones científicas (Aleixandre-Benavent, 2010). La evaluación de la investigación científica permite conocer el rendimiento de esta actividad y su impacto en la sociedad, por lo que viene a constituirse en un elemento importante en la decisión de políticas públicas y la adecuada asignación de los recursos para la investigación y desarrollo.

Para medir la producción científica de un individuo, institución o país se utiliza la bibliometría, que es una metodología que recurre a parámetros como el factor de impacto, la citación de los artículos publicados, entre otros. Los análisis bibliométricos son importantes para evaluar actividades de investigación y su contribución a actividades específicas del país, lo que además permite tomar decisiones en campos de investigación determinados (Pu y otros, 2016; Coccia y Rolfo, 2007). Por lo tanto, los análisis de producción científica explican el estado de la ciencia de una manera más real, buscando conocer el comportamiento de la ciencia, las áreas temáticas de trabajo, las principales revistas, autores más productivos, los frentes de investigación, entre otros. Muchos de los estudios realizados se basan en la información publicada en las bases de datos comerciales, mismas que recogen parte de la producción científica generada por los países (Ríos y Herrero, 2005).

Ecuador es un país de economía pequeña y emergente, que depende en gran parte del petróleo, sin embargo, en la actualidad se encuentra haciendo esfuerzos considerables para ubicarse a la altura de países creadores de conocimiento de calidad (Álvarez-Muñoz y Pérez-Montoro, 2015). La evolución de la producción científica en Ecuador ha seguido un patrón similar a la de otros países latinoamericanos, en donde aspectos como la falta de incentivos gubernamentales ha dado lugar a un escenario de escasa producción científica (Brunner y Ferrada-Hurtado, 2011). No obstante, en los últimos años, el gobierno ecuatoriano a través de la generación de leyes, políticas y reglamentos (por ejemplo: la Ley Orgánica de Educación Superior, el Plan Nacional para el Buen Vivir 2017-2021 y el Reglamento para la Acreditación, Inscripción y Categorización de Investigadores nacionales y Extranjeros que realicen Investiga- ción en el Ecuador), avances en las universidades, estímulos para obtener títulos de postgrado (becas), creación de programas de transferencia científica y tecnológica y el suministro de fondos para financiar proyectos interinstitucionales de investigación científica a nivel nacional e internacional, ha venido promoviendo el aumento de la producción científica (Medina y otros, 2016).

En el 2014, con el objetivo de reconocer a los investigadores del Ecuador de acuerdo a su producción científica, formación académica y trayectoria profesional, la Secretaría de Educación Superior, Ciencia, Tecnología e Innovación (SENESCYT) creó el "Reglamento para la Acreditación, Inscripción y Categorización de Investigadores Nacionales y Extranjeros que realicen Investigación en el Ecuador", incrementando el salario para investigadores de los Institutos Públicos de Investigación (IPIs). Con este Reglamento, los investigadores debían acreditarse como tal en diversas categorías como principal, agregado y auxiliar, con base en determinados parámetros para cada categoría como una formación de maestría o doctorado, experiencia en dirección de tesis, publicaciones científicas y años de participación en investigación y desarrollo.

El Instituto Nacional Autónomo de Investigaciones Agropecuarias (INIAP) es una entidad de derecho público, con personería jurídica y patrimonio propio, desconcentrada, con autonomía administrativa, financiera y técnica, adscrita al ministerio rector (Ministerio de Agricultura) de la política agraria con el objetivo impulsar la investigación científica, la generación, innovación validación y difusión de tecnologías en el sector agropecuario y de producción forestal (Yanez y otros, 2019). El 99\% de su financiamiento procede de recursos fiscales, proveniente de una inversión estatal en la investigación agropecuaria de alrededor del 0,03\% de su Producto Interno Bruto (SENESCYT e INEC, 2014). Del 2014 al 2019, esta institución contó con alrededor de 26 millones de dólares en promedio para ejecutar todas sus actividades. En cuanto a la disponibilidad de investigadores, en el periodo 2014-2019, este número ha tenido notables variaciones, entre el 2014 y 2015 el dato se mantuvo estable (209 y 203 investigadores respectivamente), para el 2016 y 2017 se observó una reducción a 131 y 137 respectivamente; en el 2018 se incrementó nuevamente y llegó a 143, pero en el 2019, la cantidad de investigadores nuevamente disminuyó, esta vez a 111 investigadores.

Además, desde sus inicios, el INIAP ha desarroIlado material genético mejorado y otras tecnologías para el manejo de la producción agropecuaria en el Ecuador (Sánchez y Zambrano, 2019), cuyos 
resultados los ha venido plasmando en publicaciones técnicas como dípticos, guías de manejo de cultivo, publicaciones misceláneas, manuales, entre otros. Además de la producción técnica, desde el año 2014, el Instituto ha tenido un aumento sostenido en su producción científica; sin embargo, no existe un estudio detallado que analice la evolución y situación de la producción científica del INIAP. Por esta razón, el objetivo de la presente investigación fue realizar un análisis de la producción científica del INIAP durante el periodo 2014-2019, que permita conocer el estado de la investigación, su rendimiento, impacto en la comunidad científica, visualizar la difusión de los resultados de investigación mediante documentos indexados en revistas científicas y generar recomendaciones de política institucional.

\section{METODOLOGÍA}

Este estudio analizó las publicaciones técnicas y científicas realizadas por INIAP entre los años 2014 y 2019. Para analizar las publicaciones técnicas se consideraron manuales, publicaciones misceláneas, boletines (técnicos y divulgativos), trípticos o dípticos y guías de aprendizaje. Los datos provinieron de las siguientes fuentes:

- Google scholar (https://scholar.google.com/)

- Repositorio INIAP (https://repositorio.iniap. gob.ec/)

Para analizar las publicaciones científicas con y sin colaboración interinstitucional, nacional o internacional, se consideraron artículos y notas científicas, y capítulos de libros publicados en bases de datos indexadas, los datos provinieron de las siguientes fuentes:

- Scopus (https://www.scopus.com/)

- Plumb analytics (https://plumanalytics.com/)

- Google scholar (https://scholar.google.com/)

- Scielo (https://www.scielo.org/es/)

- $\quad$ Springer (https://www.springer.com/la)

- Science Direct (https://www.sciencedirect. $\mathrm{com} /) ; \mathrm{y}$,

- Páginas de la revista en la que se publicaron los artículos.

La metodología utilizada para la búsqueda de información fue similar a la empleada por Castillo y Powell (2019), con modificaciones, ya que, para las publicaciones técnicas, nuestra estrategia de búsqueda consistió en identificar las publicaciones generadas durante el periodo 2014-2019 en el repositorio INIAP y registrar las visualizaciones y descargas, por año. En las publicaciones científicas la estrategia de búsqueda consistió en identificar las publicaciones con, al menos, un autor perteneciente al INIAP, en calidad de autor principal o coautoría con investigadores de otros países, y clasificarlas de acuerdo al índice SCImago Journal \& Country Rank (SJR), que es un factor de medición que establece la influencia científica de las revistas basándose en el recuento de citas obtenidas por cada publicación, además en este grupo se registró el factor de impacto de las revistas a las que perteneció cada publicación. Es así que la base de datos se dividió en dos grupos, el primero conformado por aquellas publicaciones que poseían el índice SJR y el otro grupo por aquellas publicaciones que no poseían este índice, pero que están indexadas en Scielo, Latindex y otras bases de datos. La información se depuró de forma manual mientras se comprobaba visualmente las citas y el nombre de cada revista científica, todo esto con el fin de evitar errores de transcripción.

Con la información obtenida, se usaron estadísticas descriptivas (media, mediana, percentiles, gráficos de tendencia, histogramas) e indicadores como artículos por año, artículos per cápita, artículos por cada cien mil dólares invertidos, citas por año, citas por autor, citas por artículo, tasa de crecimiento anual compuesta y medida de Salton.

El aumento de la producción científica del INIAP durante el periodo 2014-2019 se calculó a través del total de publicaciones totales por año. Se utilizó la tasa de crecimiento anual compuesta (CARG) para medir el crecimiento científico a través del tiempo (Hassan y otros, 2015)

$$
C A G R=\sqrt[n]{(P 2 / P 1)}
$$

Donde $P 1$ corresponde al número de publicaciones del INIAP en el año 2014; $P 2$ es el número de publicaciones del INIAP en el año 2019; y, $n$ el número de años del periodo (6 años).

Se analizó el nivel de colaboración del INIAP con instituciones ecuatorianas, países de América Latina, Norteamérica, Europa y Otras regiones (Asía, África y Oceanía), para valorar la importancia relativa a la actividad científica conjuntamente con INIAP. Para determinar el nivel de colaboración entre INIAP y otros países en el área de Agricultura y Ciencias Biológicas, se utilizó la medida de Salton (Castillo y Powell, 2019):

$$
S=\frac{P C_{1.2}}{\sqrt{P C_{1} * P C_{2}}}
$$


Donde $P C_{1.2}$ corresponde al número de publicaciones conjuntas entre el INIAP y otro país; $P C_{1}$ es el número de publicaciones totales del INIAP; y, $P C_{2}$ corresponde al número de publicaciones totales del país colaborador.

Para los análisis de región se agrupó las publicaciones de acuerdo a las siguientes regiones: Ecuador, Latinoamérica, Norteamérica, Europa y otras regiones del mundo (Asia, África y Oceanía) que corresponde a publicaciones con colaboración internacional en menor escala con otros países no incluidos en las regiones mencionadas anteriormente. Además, se consideraron todas las combinaciones de estas regiones: Ecuador + Latinoamérica; Ecuador + Norteamérica; Ecuador + Europa; Latinoamérica + Europa; Latinoamérica + Norteamérica; Norteamérica + Europa; Ecuador + Latinoamérica + Europa; Latinoamérica + Norteamérica + Europa; Ecuador + Norteamérica + Europa, también consideramos en la categoría 'otras regiones del mundo'. La combinación de regiones nos sirvió para clasificar las publicaciones del INIAP de acuerdo a la colaboración internacional y establecer las regiones que han sido más productivas con el INIAP en el periodo 2014-2019.

Además, se analizó la distribución del porcentaje de publicaciones científicas según las áreas de investigación establecidas en el Plan Estratégico Institucional 2019-2022 del INIAP (Yanez y otros, 2019) durante el periodo en estudio. Un punto importante analizado en este estudio fue la participación de la mujer en la producción científica del INIAP; para lo cual se calculó el porcentaje de participación en publicaciones científicas como autora principal o en coautoría.

Adicionalmente con el fin de conocer el orden de los autores de las publicaciones y la región a la que pertenecen, las publicaciones se clasificaron por la afiliación del primer autor y afiliación del autor corresponsal.

\section{Modelo de regresión}

Se utilizó un modelo de regresión para describir la relación entre el 'número de citas' como variable respuesta con un conjunto de variables $X_{1^{\prime}} X_{2}$ $X_{3} \ldots, X_{p}$ que representan las variables independientes con alguna relación lineal con la variable dependiente. Es habitual que algunas variables usadas en un modelo de regresión múltiple no estén asociadas con la variable respuesta por lo tanto es necesario llevar los términos de regresión a un valor cero para obtener un modelo que sea fácil de interpretar (MuIler y Feterman, 2002), para ello se utilizó la técnica de mínimos cuadrados en donde se calcularon los coeficientes y además se incorporó un procedimien- to de selección por pasos para obtener el modelo lineal (Markovsky y Van Huffel, 2007).

En este sentido, el modelo de regresión puede ser expresado con la siguiente fórmula:

$$
Y=\beta_{0}+\beta_{0} X_{1}+\ldots+\beta_{p} X_{p}+\varepsilon
$$

Donde, $Y$ es el 'número de citas'; $\beta$ son los coeficientes de regresión; $y, X$ son las variables independientes que explican el modelo estadístico. Además, para cumplir con los supuestos del modelo de regresión (Marqués de Sá, 2007), la variable dependiente se transformó a logaritmo natural más 1 .

\section{RESULTADOS Y DISCUSIÓN}

En cuanto a los impactos generados por el INIAP, se sabe que cada dólar que el Estado invierte en el desarrollo de nuevas tecnologías agropecuarias en el INIAP genera 40 veces su valor, además los productores adoptan éstas tecnologías e incrementan sus ingresos en alrededor de un $32 \%$ (Tinoco, 2018). En cuanto a la adopción de tecnologías, el material genético y las tecnologías o prácticas de manejo desarrolladas por el INIAP han sido adoptadas en un promedio de $37 \%$, en donde el material genético tuvo un porcentaje de adopción de $38 \%$, tecnologías de manejo de cultivo $35 \%$ y prácticas de manejo 38 \% (Sánchez y Zambrano, 2019). En términos de rubros agrícolas, la adopción de las tecnologías generadas por INIAP por parte de los agricultores de café fue un $23 \%$, cacao $29 \%$, cereales $41 \%$, raíces y tubérculos $27 \%$, frutas $21 \%$, leguminosas $12 \%$, granos andinos $60 \%$ y oleaginosas $37 \%$ (Naranjo, 2017). El INIAP aporta en alrededor del $16,5 \%$ al PIB agropecuario expresado en toneladas métricas y tiene influencia directa en cerca del 20,9 \% del empleo que se genera en el sector agrícola. Las actividades del Instituto también influyen en un $26,6 \%$ de la superficie dedicada a cultivos (Tinoco, 2018). La diversificación de los sistemas productivos mediante la implementación de chacras, la capacitación en el manejo y el uso de la agro biodiversidad aumentan los ingresos medios mensuales de los productores hasta en un 29,5\%; también INIAP ha generado alternativas tecnológicas sostenibles para mejorar la productividad en animales bovinos a través de la nutrición, sanidad y manejo, las cuales pueden lograr un incremento en el volumen de producción de hasta $122 \%$; incremento del 40 al $60 \%$ en producción de forrajes (Tinoco, 2018). Desde la creación del Instituto, hasta la actualidad, el instituto ha generado 274 materiales mejorados entre variedades, híbridos y clones, y se ha desarrollado cerca de 470 alternativas tecnológicas de producción y agregación de valor; la tasa de generación de tecnologías ha cambiado en los últimos años, durante el pe- 
ríodo 1960-2012 se desarrollaron en promedio 7,4 tecnologías por año, mientras que en la actualidad la tasa es de 15,2 tecnologías año (Tinoco, 2018). En todos los rubros en que trabaja el INIAP, los rendimientos de los materiales mejorados y tecnologías generadas por el instituto son superiores a los rendimientos nacionales, y en algunos cultivos los superan por más del $100 \%$; el Instituto también conserva la diversidad genética de interés para la alimentación y la agricultura: ex situ se mantiene más de 28 mil accesiones e in situ ha establecido siete centros de Bioconocimiento y Desarrollo Agrario a nivel Nacional (Tinoco, 2018). Muchos de los productos de las investigaciones se encuentran en publicaciones técnicas que se encuentran disponibles permanentemente en el repositorio digital del INIAP, mismo que es actualizado constantemente; además, de manera física, estas publicaciones técnicas son socializadas a agricultores, técnicos y estudiantes en eventos de capacitación organizados por las estaciones experimentales, es así que durante el periodo 2014-2019 el promedio/año de asistentes a estos eventos de capacitación fue de 11.000 personas aproximadamente.

Por otro lado, una temática de relevancia para el Instituto es la incorporación de valor agregado a la producción agropecuaria (Zambrano y otros, 2018). Existen casos exitosos del aporte del INIAP al desarrollo de la agroindustria ecuatoriana: 1) La marca "Kiwa", es una marca global de chips de vegetales Premiun que se comercializan en más de 20 países, nace de un proyecto Fontagro en el año 2011, en el cual se generan las variedades mejoradas INIAP- Puca Shungo e INIAP-Yana Shungo, con las cuales se realiza una vinculación de los productores a mercados diferenciados, el INIAP aportó con el desarrollo de las variedades mejoradas de papa, disponibilidad de semilla y capacitación a productores vinculados a la provisión de materia prima a la empresa INALPROCES. 2) Desarrollo de snacks a base de chocho, para lo cual en el año 2017 se firmó una carta de entendimiento con el Grupo Grandes, luego se iniciaron los procesos para el desarrollo de productos a base de chocho, Grandes Foods impulsó emprendimientos productivos de chocho especialmente en la parroquia Chugchilan, provincia de Cotopaxi, con lo cual permitió que sus productos sean comercializados en uno de los principales supermercados del país: Supermaxi; el INIAP aportó con el desarrollo y adaptación de tecnología para la producción de chocho crocante, harina de chocho, chocho crocante con cubierta de canela y chocolate. 3) Investigación y desarrollo de productos a base de Amaranto, gracias al desarrollo de variedades mejoradas de amaranto como el caso de INIAP-Alegría, la empresa The Andean Secret inició el proceso de desarrollo de productos, trabajó con comunidades indígenas en el sistema "fair trade", Amati es la primera bebida de Amaranto a nivel mundial empacada en tetrapack, el INIAP aportó con el desarrollo de la variedad mejorada de amaranto y con disponibilidad de semilla. 4) Validación de variedades mejoradas de cebada INIAP. Dentro de los procesos utilizados por la marca Latitud Cero se utilizó la variedad mejorada de cebada INIAP-Cañicapa en las mezclas con otras cebadas malteras, es la primera cerveza artesanal producida en Ecuador y se comercializa actualmente en Supermercados del país. Además, el INIAP aportó con el desarrollo de la variedad mejorada de cebada y con disponibilidad de semilla.

\subsection{Análisis Bibliométrico}

En el periodo 2014-2019, la producción técnica del INIAP fue de 107 de publicaciones. La Figura 1 muestra que en el 2014 el número de publicaciones técnicas fue de 29, luego en al año 2015 disminuye a 16 publicaciones para mantener una tendencia similar hasta el 2019 (15 publicaciones técnicas). Esta disminución en el número de publicaciones técnicas se debe a que en este periodo los investigadores de INIAP generaron un mayor número de publicaciones científicas, efecto ligado al proceso de categorización de los investigadores ante la SENESCYT.

El promedio de citas en google scholar durante el periodo en estudio fue de 0,6 citas por publicación, en el año 2017 se alcanzó el valor máximo con 1,3 citas por publicación.

A la fecha del análisis, se observó que las publicaciones del año 2014 disponen del mayor número de visualizaciones y descargas (Figura 2), debido a su tiempo de permanencia en el repositorio digital de la institución. Este aspecto estaría relacionado a la temática (rubro agrícola) del documento, siendo así que las publicaciones técnicas sobre los cultivos de palma aceitera, frutales (cítricos, aguacate y mora), cacao, granos andinos, maíz y papa fueron las más descargadas y/o visualizadas durante el periodo 2014 al 2019.

La producción científica del INIAP fue de 246 publicaciones, de las cuales el $64 \%$ de los documentos se publicaron en idioma inglés y el $36 \%$ en español. La Figura 3 muestra el total de la producción científica del INIAP en el periodo 2014 al 2019. El $62 \%$ de las publicaciones estuvieron indexadas en Scopus, $24 \%$ en Latindex, $8 \%$ en Scielo y $6 \%$ en otras bases indexadas. Del total de publicaciones científicas indexadas a la base Scopus, $47 \%$ tuvieron un índice SJR entre 0 y $1 ; 12 \%$ entre 1 y $2 ; 2 \%$ entre 2 y $3 ;$ y, el $1 \%$ de las publicaciones científicas tuvieron un índice SJR entre 3 y 4. 
Figura 1. Número y promedio de citas en google scholar, de las publicaciones técnicas en el INIAP, periodo 2014-2019

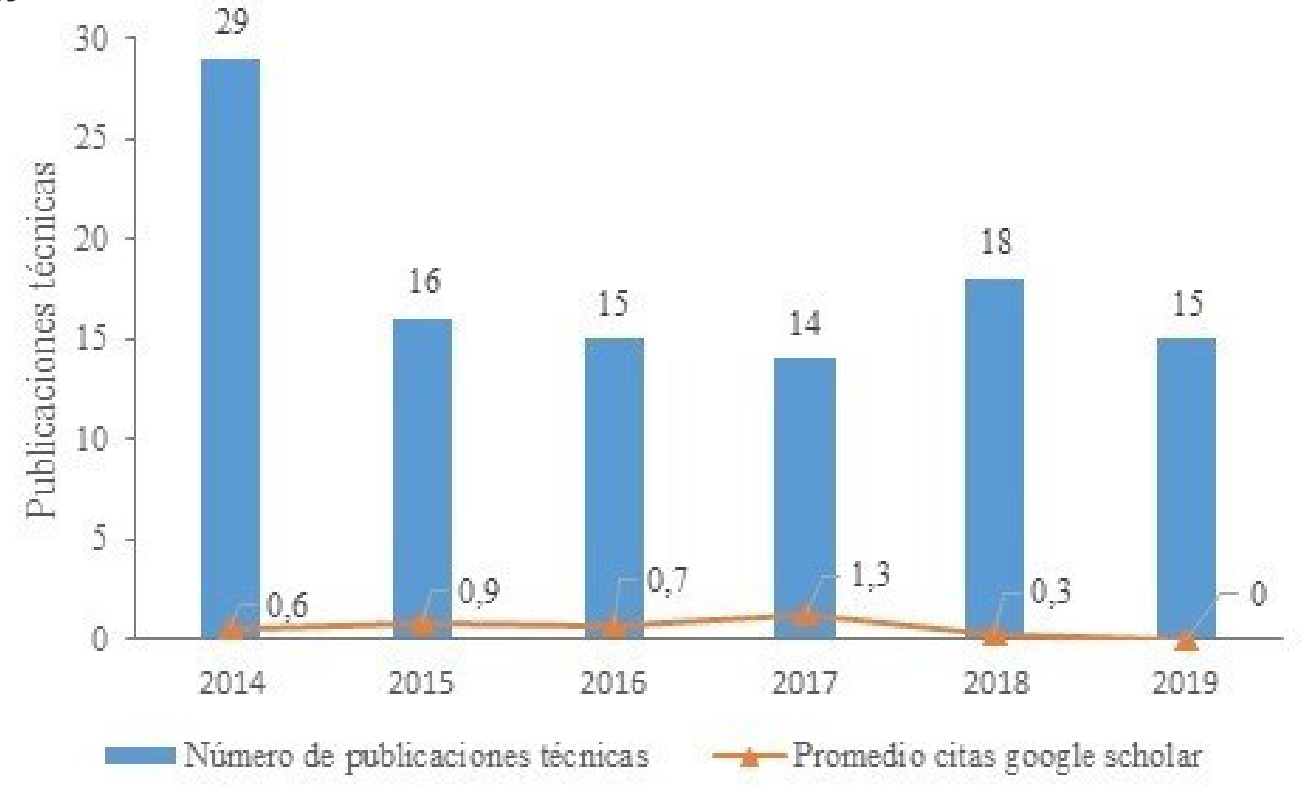

Figura 2. Número de visualizaciones y descargas de las publicaciones técnicas del INIAP en el repositorio, por año de publicación

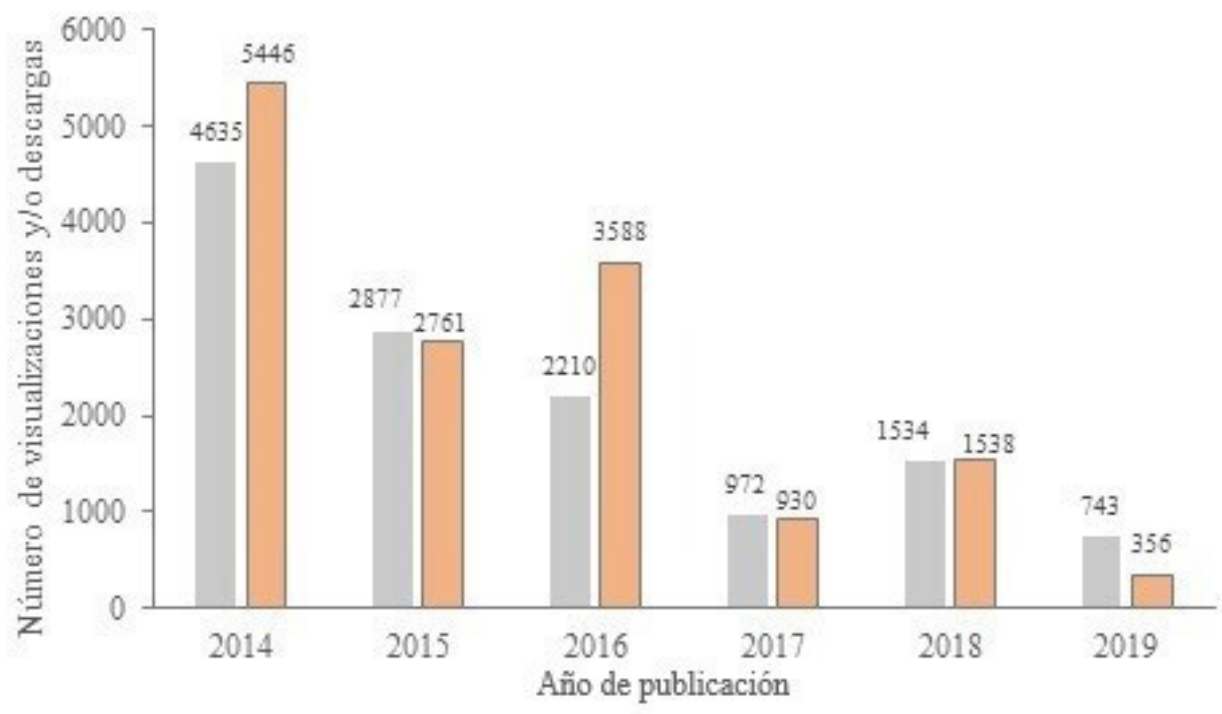

- Visualizaciones en repositorio INIAP $\quad$ Descargas en repositorio INIAP

Del total de publicaciones con índice SJR, en la Figura 4 se observa que el 2014 fue el año que tuvo el mayor promedio del índice $\operatorname{SJR}(1,1)$ y a la vez el mayor factor de impacto de sus revistas $(2,62)$; en cambio en el 2019 se observó una disminución de los promedios de los índices mencionados $(0,65$ y 1,7 respectivamente), debido a que existen publicaciones científicas que no ingresaron al análisis porque cuando se recopiló la información (septiembre 2019), se encontraban en proceso de revisión para aceptación y publicación en la revista. Además, se realizó un análisis de correlación entre el índice SJR y el factor de impacto en donde la correlación entre estos dos indicadores fue significativa $r=0,89$ ( $p<0,001)$, por lo cual los investigadores del área agropecuaria podrían basarse en cualquiera de estos dos índices al momento de seleccionar una revista de influencia científica. 
Figura 3. Producción de publicaciones científicas en el INIAP, periodo 2014-2019

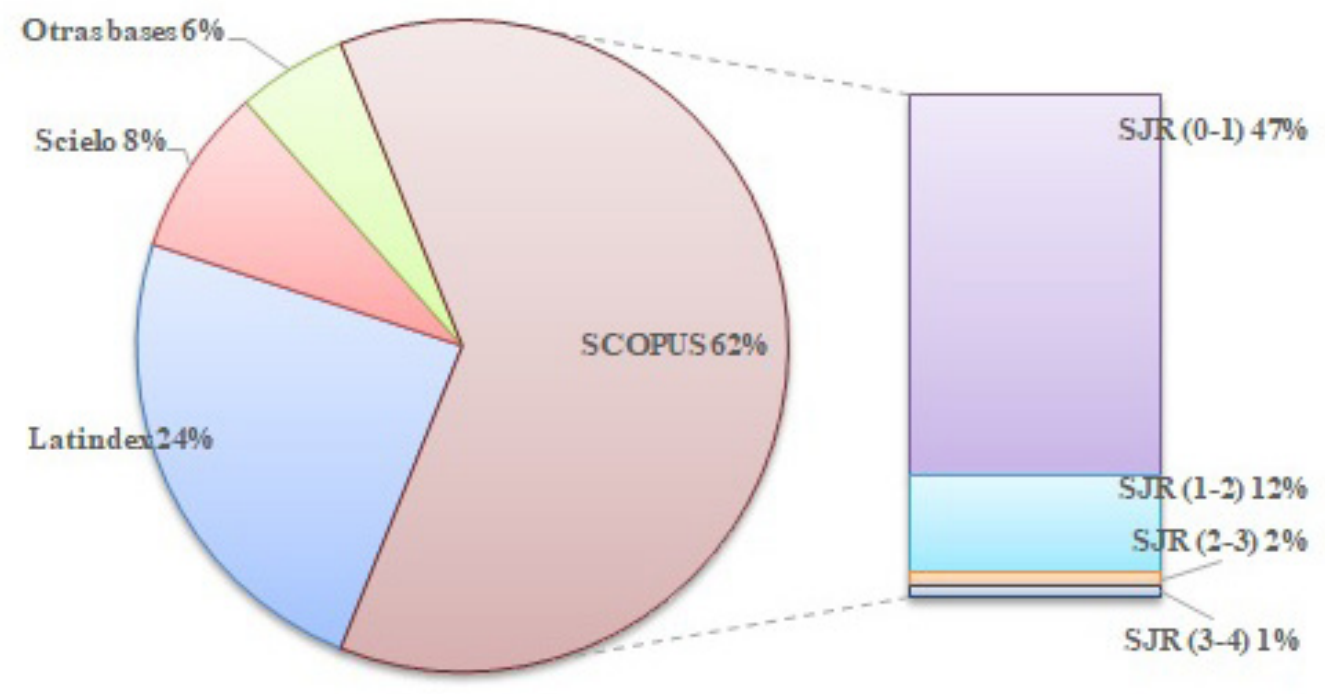

Figura 4. Evolución del índice SJR y factor de impacto, en revistas científicas a las que las publicaciones científicas del INIAP se encuentran indexadas, en el periodo 2014-2019

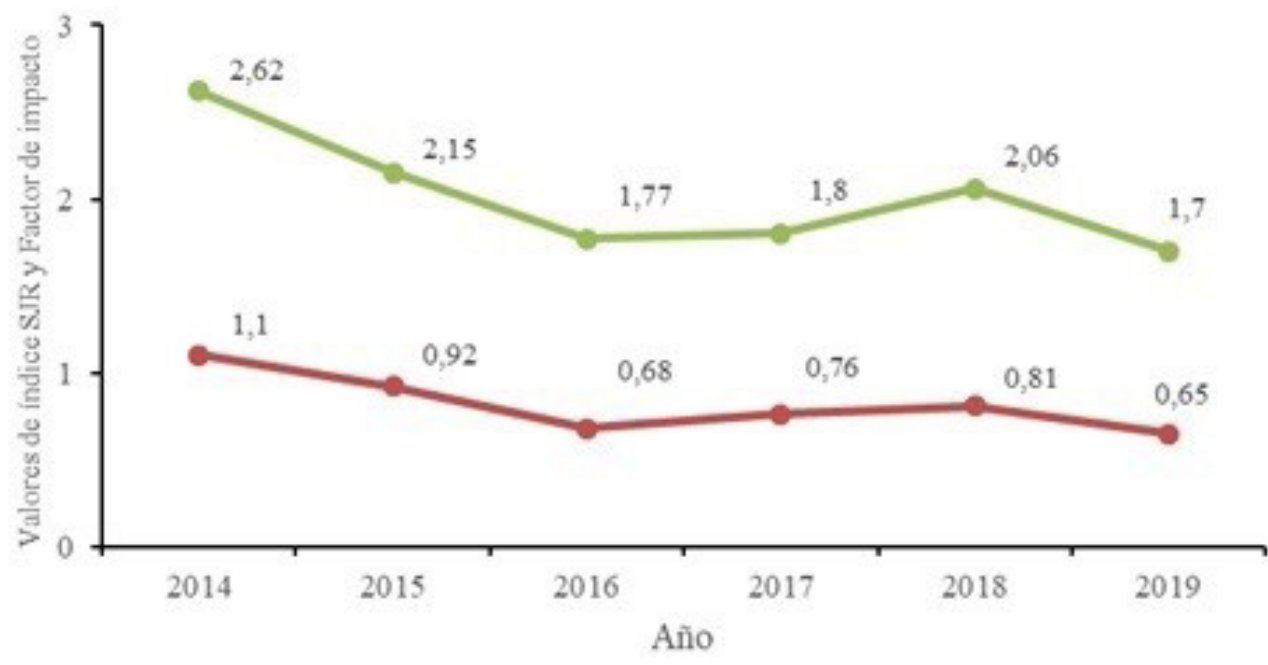

-SJR $\quad$ - Factor de Impacto

Durante el período de estudio se obtuvo un valor promedio del índice SJR de 0,82 y un factor de impacto promedio de 2,01. El factor de impacto sirve para comparar revistas y evaluar la importancia relativa de una revista concreta dentro de un mismo campo científico, mientras que SJR establece que la calidad y reputación de una revista científica tienen un impacto directo sobre el valor de la citación, esto significa que la cita de una fuente con SJR alto tiene más valor que la cita de una fuente con un SJR menor. En términos del índice SJR, el resultado promedio obtenido en este estudio es más alto que el valor en Latinoamérica en el área de agricultura y ciencias biológicas (SJR 0,763); sin embargo, es inferior al valor a nivel mundial (SJR 7,04).

Se observó también un porcentaje considerable de publicaciones en la base Latindex (24\%), puesto que varias investigaciones producen resultados de carácter regional. Finalmente, se obtuvo un porcentaje bajo de publicaciones indexadas en la base de Scielo ( $8 \%$ ); sin embargo, consideramos que este porcentaje aumentará con el transcurso del tiempo debido que SciELO Ecuador es parte de las políticas de SENESCYT de fomento a la investigación y difusión de sus resultados. 
El aumento de publicaciones científicas del Instituto entre el año 2014 y 2019 se calculó dividiendo el número de publicaciones del año 2019 por el número de publicaciones del 2014 y correspondió a 2,9 veces, valor mayor al promedio de Latinoamérica en este periodo (1,2 veces). La tasa de crecimiento anual compuesta (CARG) del INIAP entre los años 2014 y 2019 fue de 23,7\%, lo que indica que el Instituto ha tenido un considerable crecimiento en la producción científica, tomando en cuenta que el CARG promedio para los países de Latinoamérica fue de $4,3 \%$ en el mismo periodo de tiempo. Según Castillo y Powell (2019), el número de publicaciones científicas en Latinoamérica se duplicó en el período comprendido entre el año 2006 (68.706 publicaciones) y 2015 (121.502 publicaciones), en cambio, en nuestro periodo de estudio, el número de publicaciones científicas para Latinoamérica en el año 2014 fue de 25.910 documentos; mientras que para el año 2019 se incrementaron a 30.256 publicaciones, es decir un incremento de un $17 \%$. Estos datos coinciden con el hecho de que Latinoamérica al igual que Ecuador, han aumentado el número de investigadores en los últimos años, reflejándose esto en el incremento de la producción científica (Catanzaro y otros, 2014; Organización de las Naciones Unidas para la Educación, la Ciencia y la Cultura, 2015; Kreimer y Heimer, 2018).

Por otro lado, el producto interno bruto (PIB) de cada país es determinante debido a que el número de publicaciones científicas se incrementa conforme aumenta el PIB, este incremento se puede justificar debido a que un mayor PIB involucra una mayor inversión en herramientas para investigación y producción intelectual (Álvarez-Muñoz y Pérez-Montoro, 2015). Según Quinde-Rosales y otros (2019), la evolución del gasto en Ciencia y Tecnología y del Producto Interno Bruto en el Ecuador presentaron una tendencia positiva de crecimiento, similar a la tendencia de América Latina y el Caribe durante el periodo 1990-2015. Desde el 2009 el gasto en Ciencia y Tecnología mostró un crecimiento del $122 \%$ entre el 2009 (172,48 millones de dólares) y 2014 (382,92 millones de dólares), mientras que el gasto en Investigación y Desarrollo subió de 145,95 millones de dólares en el 2009 a 259,81 millones de dólares en el 2014 (SENESCYT, 2014). Según la Red de Indicadores de Ciencia y Tecnología Iberoamericana e Interamericana, (2018), Estados Unidos y China son los países que más invierten en ciencia y tecnología con $2,7 \%$ y $2,1 \%$ de PIB de cada país respectivamente. En la región, Colombia invierte el 0,23 \% y el Ecuador invierte $0,26 \%$. La inversión ecuatoriana se distribuye en el sector productivo $58 \%$, en el sector gubernamental $24 \%$, en el sector educativo $12 \%$ y la inversión privada directa $6 \%$. Junto a esta ten- dencia de crecimiento en inversión, es evidente el aumento en la producción científica ecuatoriana, así lo confirman los datos obtenidos por Castillo y Powell (2019). Además, en el 2017 Ecuador ocupó el puesto 66 entre 230 países en la producción mundial de artículos científicos.

En la Figura 5, se observa que de los 246 documentos científicos publicados por INIAP en el periodo 2014-2019, la producción máxima se alcanzó en el año 2017, observándose un incremento de 3,1 veces en comparación con el año 2014 y el mismo valor en comparación con el 2015; mientras que, en el año 2018, esta tendencia decrece a 1,4 veces, finalmente en el año 2019 el número de publicaciones aumenta nuevamente en 1,3 veces en comparación con el año 2018. Este comportamiento de aumento en la producción científica en el Ecuador ha sido observado también por Castillo y Powell (2019), puesto que, en un periodo de diez años, reportaron un aumento de 1,5 veces en la producción científica entre los años 2004 y 2015. Resultados que permiten inferir que la producción científica en el Ecuador continua en aumento de manera significativa.

Por otra parte, la media de la cantidad de autores por publicación en el período en estudio fue de 7 , valor que es superior a 4,08 autores por publicación reportado por Bravo-Vinaja y Sanz-Casado (2008). En nuestro estudio, el mayor dato de este indicador bibliométrico se observa en el año $2015(8,2)$ y el menor en el año $2014(5,3)$. Estas cifras indican el alto nivel de cooperación que tiene el Instituto para la elaboración de publicaciones científicas. El promedio del número de artículos per cápita durante nuestro periodo de análisis fue de 0,3 ; en donde, el año 2019 presenta el valor más alto $(0,50)$ y los años 2014 y 2015 presentaron el menor vaIor $(0,09)$. El promedio del número de artículos por cada cien mil dólares invertidos durante el periodo 2014-2019 fue de 0,5, es así que en los años 2017 y 2019 se presenta el valor más alto $(0,75)$ mientras que en el año 2015 se observó el menor número de artículos por cada cien mil dólares invertidos $(0,12)$.

La citación de las publicaciones (Figura 6) es un aspecto muy estudiado como indicador de impacto (Thelwall, 2018). Este factor refleja el reconocimiento y la utilidad que está teniendo el documento científico generado (Cañedo-Andalia, 1999, González y otros, 2016). Además, la citación permite identificar el número de citas por año, por artículo y por autor. Los artículos publicados entre el 2016 y 2017 cuentan con la mayor cantidad de citaciones (360 y 326 respectivamente); este comportamiento tiene relación directa con la mayor cantidad de publicaciones disponibles en esos años. Por otro lado, tomando en cuenta el índice de 
Figura 5. Análisis de artículos por año, artículos per cápita, artículos por cada cien mil dólares invertidos y número de autores por publicación, de las publicaciones científicas del INIAP durante el periodo 2014-2019

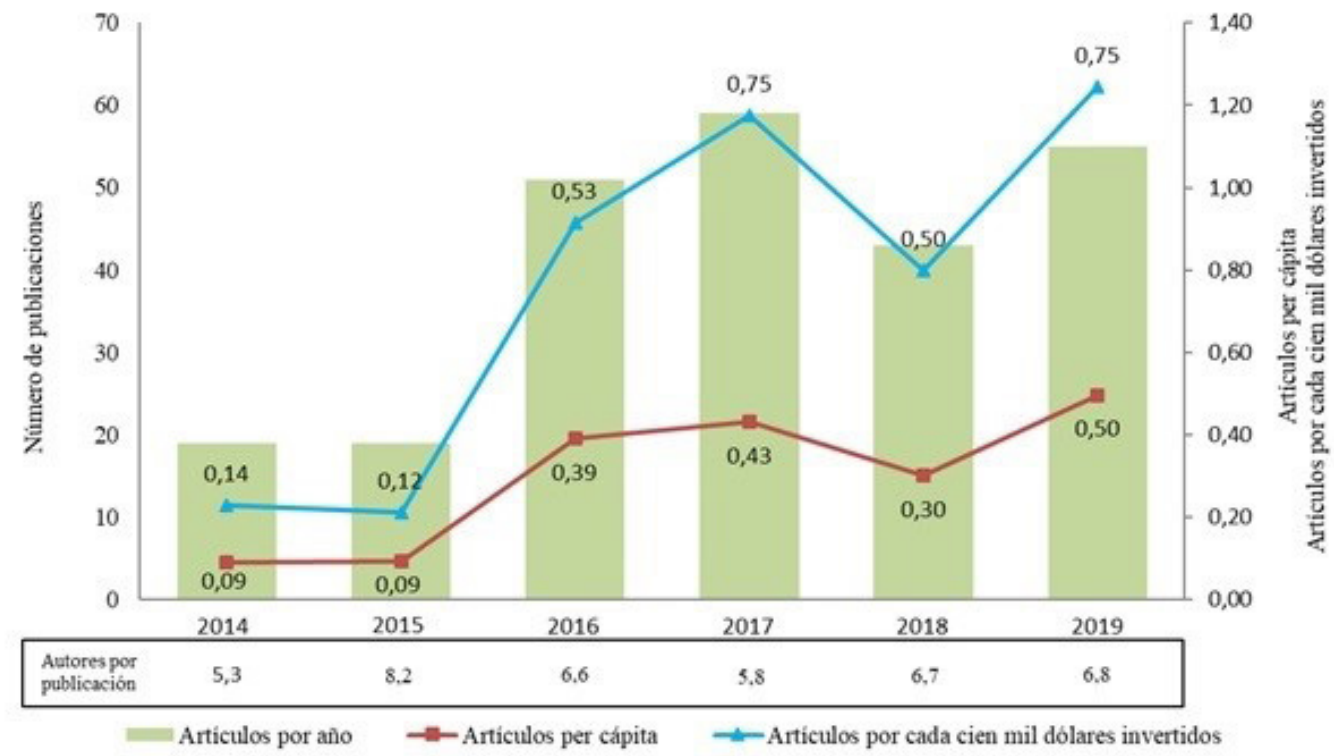

citación por artículo, se observó que los documentos individuales más citados fueron los publicados en el año $2014(14,9)$ y los menos citados en el año $2019(0,5)$; siendo esta tendencia explicada por la antigüedad del artículo ya ha tenido mayor tiempo disponible para consulta. En el periodo de análisis, la media de citas por autor fue de 1,3; sin embargo, los artículos científicos publicados en el año 2014 son los que mayor citación por autor presentaron (4). Estos resultados corroboran lo indicado por Sánchez Pereyra y otros, (2015) ; y, De Granda-Orive y otros (2013) quienes mencionan que el número de citas de los documentos publicados en los años anteriores es más alto.

La Figura 7 indica el porcentaje de publicaciones de acuerdo a las áreas de investigación de INIAP (Zambrano y otros, 2018). Se observa que el $69 \%$ de las publicaciones científicas del INIAP en el periodo 2014-2019 se encuentran enmarcadas en el área de investigación de 'incremento de la productividad del sector agropecuario', el 14\% de documentos se alinean al área de 'manejo y conservación de los recursos naturales de interés para la agricultura y alimentación' y $11 \%$ están enmarcadas en el área de 'incorporación de valor agregado a la producción agropecuaria'. Sin embargo, por especificidad de la temática adicionalmente separamos las publicaciones sobre economía agrícola y cambio climático que corresponden a un $6 \%$.

Con el fin de resolver problemas puntuales en diferentes áreas de interés, los países con baja producción científica focalizan su investigación en campos determinados (Pouris y Ho, 2014; Hassan y otros, 2015). El mayor porcentaje de publicaciones científicas de INIAP (69\%), se alinea al área de investigación de 'incremento de la productividad del sector agropecuario', en donde el enfoque de ésta área de investigación está en el mejoramiento genético y la agrobiotecnología como estrategias para generar materiales mejorados adaptados a las condiciones agroclimáticas, lo cual se complementa con procesos de investigación, desarrollo e innovación en el manejo integrado de cultivos y ganado (Yanez y otros, 2019).

La Figura 8 muestra la participación de la mujer en la producción científica del INIAP. Para la sociedad ecuatoriana, son muy poco conocidos los hallazgos importantes en la ciencia por parte de las mujeres científicas e investigadoras ecuatorianas (Pessina, 2019). En este estudio, se pudo observar un porcentaje de participación constante y similar entre el 2014 y 2019, observándose que el rol de la mujer en la investigación es permanente en el INIAP; sin embargo, su porcentaje de participación estaría relacionado el número de investigadoras mujeres en la institución. En el año 2014, el 37\% de las publicaciones científicas de INIAP tienen a una investigadora como autora principal; mientras que el $27 \%$ de los autores y coautores de las publicaciones de ese año fueron mujeres. En el 2019, el $31 \%$ de las publicaciones científicas poseen a una investigadora como autora principal; mientras que el $18 \%$, de la población total de autores y coautores en este año, fueron mujeres. 
Figura 6. Análisis de citas por año, citas por autor y citas por artículo de las publicaciones científicas del INIAP durante el periodo 2014-2019

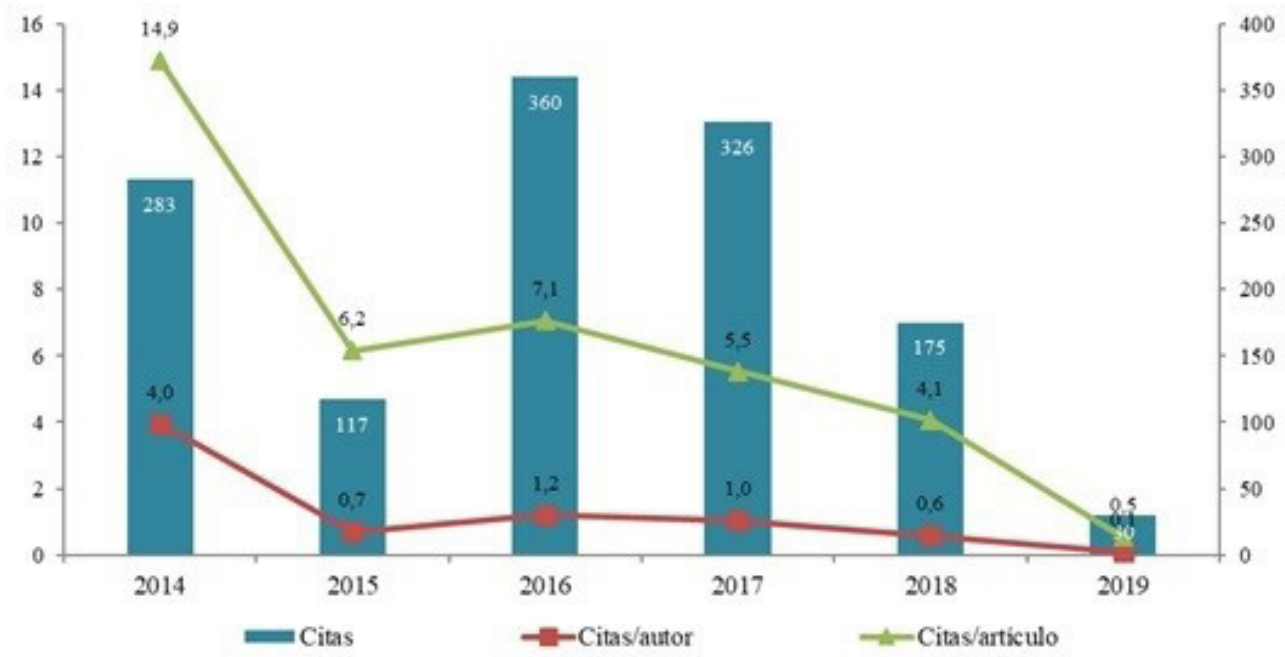

Figura 7. Porcentaje de publicaciones indexadas de acuerdo al área de investigación según el PEI del INIAP, durante el periodo 2014-2019

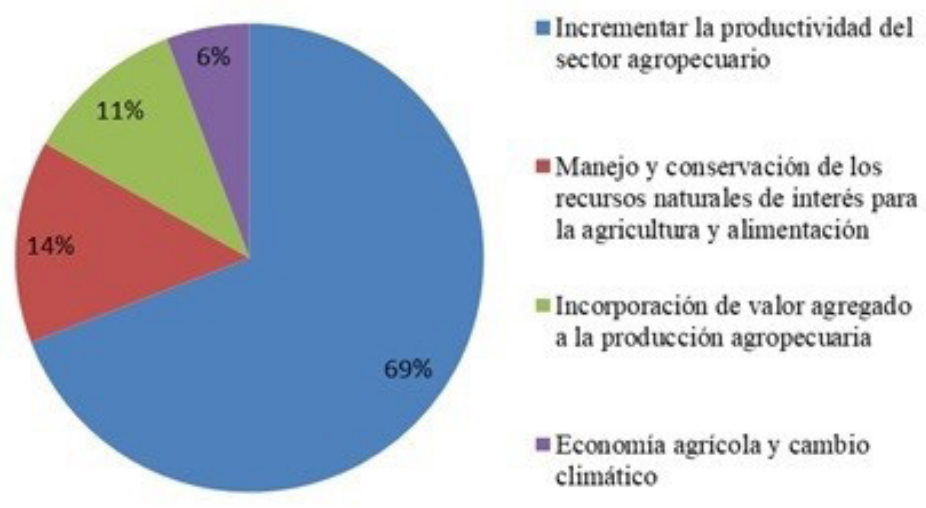

Figura 8. Participación de la mujer en la producción científica del INIAP en el periodo 2014-2019. En paréntesis se indica el número de artículos publicados por año

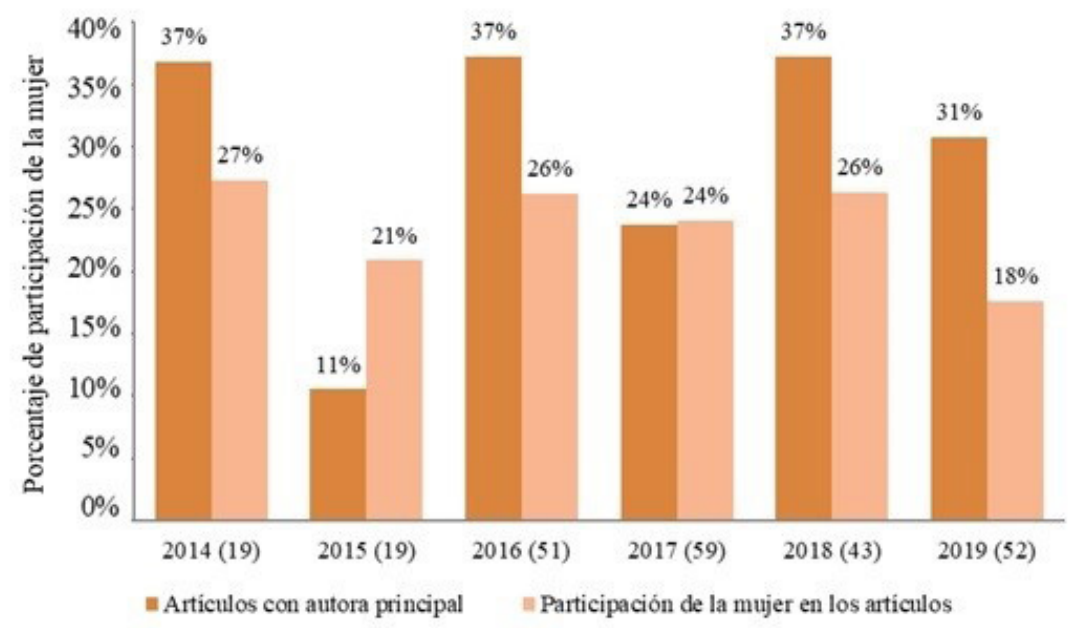


La población entre hombres y mujeres de los países iberoamericanos está fraccionada en partes prácticamente iguales, en donde el $50,6 \%$ de la población es femenina, además se observa que la tercera parte de los países iberoamericanos, revelan una cierta uniformidad de género, con porcentajes de participación femenina en la investigación entre $48 \%$ y $53 \%$ (Red de Indicadores de Ciencia y Tecnología Iberoamericana e Interamericana, 2018). El número de mujeres investigadoras en el Ecuador es menor al promedio de los países de Latinoamérica y el Caribe (44\%) (Organización de las Naciones Unidas para la Educación, la Ciencia y la Cultura, 2015). En la región, el país que cuenta con el mayor porcentaje de investigadoras en relación al total de quienes investigan es Venezuela (61\%), en cambio Perú es el país con menor número de investigadoras (32\%) (Red de Indicadores de Ciencia y Tecnología Iberoamericana e Interamericana, 2018), mientras que Ecuador posee un $35 \%$ de investigadoras (Zambrano, 2019). En el INIAP existen 16 investigadoras acreditadas, de las cuales el $38 \%$ se encuentra en la categoría 'Investigador Auxiliar 1 '; un $18,5 \%$ en la categoría 'Investigador Agregado 2'; en las categorías 'Investigador Auxiliar 2', 'Investigador Agregado 1' e 'Investigador Agregado $3^{\prime}$ se ubica un $12,5 \%$ en cada una; $y$ apenas el $6 \%$ se encuentra en la categoría 'Investigador Principal 1'. Los principales requisitos para pertenecer a las diferentes categorías de investigador, se presentan en la Tabla I. Las revistas de nivel uno son aquellas que están recogidas en las bases de datos Web of Science, o Scopus; y que correspondan a revistas situadas en el primer o segundo cuartil según el Journal Citation Reports
(JCR) o Scimago Journal Ranking (SJR); nivel dos, son aquellas que están publicadas en revistas recogidas en las bases de datos Web of Science, o Scopus; y que corresponden a revistas situadas en el tercer o cuarto cuartil según el Journal Citation Reports (JCR) o Scimago Journal Ranking (SJR); se consideran también las publicaciones de revistas indexadas en SciELO. Nivel tres, se consideran a las obras de relevancia y artículos indexados que no puedan ser incluidos en niveles superiores (Secretaría de Educación Superior, Ciencia, Tecnología e innovación, 2018)

Existen ciertas diferencias entre profesionales en el área de las ciencias e ingenierías y el número de personas que cursan carreras universitarias, que marcan desigualdades entre la cantidad de hombres y mujeres dedicadas a la investigación científica. Además, se ha demostrado discriminación hacia la mujer en el ámbito laboral, como escasa atención que los jefes dan a las investigadoras mujeres y la disminución de las perspectivas profesionales luego del primer parto (Bindé, 2005). Por otro lado, para la mujer, es difícil equilibrar las responsabilidades de una carrera profesional y las provenientes de los roles de esposa y madre, además, en la región se reportan discrepancias laborales entre profesionales de diferente sexo, estas discrepancias incluyen: tipos de contrato, escalafones, duración y remuneraciones salariales (Zubieta, 2007). Sin embargo, en la actualidad, a nivel mundial se han incrementado de manera exponencial las mujeres que han contribuido con una nueva mirada a disciplinas como la biología y biomedicina (García y Pérez, 2017).

Tabla I. Principales requisitos de las categorías de los investigadores

\begin{tabular}{|c|c|c|c|c|c|c|}
\hline Categoría & & PhD & Maestría & $\begin{array}{c}\text { Tiempo de } \\
\text { participación en } \\
\text { procesos I+D }\end{array}$ & $\begin{array}{l}\text { Publicaciones } \\
\text { indexadas* }\end{array}$ & $\begin{array}{l}\text { Observaciones a publicaciones } \\
\text { indexadas }\end{array}$ \\
\hline \multirow{4}{*}{ Principal } & 4 & $x$ & & 8 años & 20 & al menos 5 han de ser de nivel 1 \\
\hline & 3 & $x$ & & 7 años & 18 & al menos 3 han de ser de nivel 1 \\
\hline & 2 & $x$ & & 7 años & 16 & al menos 2 han de ser de nivel 1 \\
\hline & 1 & $x$ & & 6 años & 12 & al menos 1 ha de ser de nivel 1 \\
\hline \multirow{3}{*}{ Agregado } & 3 & $x$ & & 5 años & 9 & $\begin{array}{c}\text { al menos } 3 \text { han de ser de nivel } 20 \\
\text { superior }\end{array}$ \\
\hline & 2 & & $x$ & 2 años & 6 & $\begin{array}{c}\text { al menos } 2 \text { han de ser de nivel } 20 \\
\text { superior }\end{array}$ \\
\hline & 1 & & $x$ & 1 año 6 meses & 3 & $\begin{array}{c}\text { al menos } 1 \text { ha de ser de nivel } 20 \\
\text { superior }\end{array}$ \\
\hline \multirow[b]{2}{*}{ Auxiliar } & 2 & & $x$ & 1 año 6 meses & 3 & nivel 3 o su equivalente \\
\hline & 1 & & $\mathrm{x}$ & 1 año & 1 & nivel 3 o su equivalente \\
\hline
\end{tabular}

*circulación internacional como autor o coautor 
Los países que más han colaborado bilateralmente con INIAP en la elaboración de publicaciones científicas, durante el periodo 2014-2019 en orden decreciente son: Estados Unidos, España, México, Francia, Brasil, Italia, Australia, Colombia, Canadá y Dinamarca. La cooperación interinstitucional e internacional resulta trascendental para la generación de documentos científicos en el INIAP. Los mayores beneficios de colaboración internacional son recibidos por países con producción científica relativamente baja, en comparación con países con mayor producción científica (Boshoff, 2010). En el período de análisis, el $84 \%$ de publicaciones científicas fueron elaboradas con colaboración interinstitucional (Tabla II). El INIAP ha logrado producir $15,9 \%$ de documentos científicos sin cooperación de otras instituciones, $54 \%$ con cooperación internacional, mientras que el 30,1\% con instituciones del Ecuador. El porcentaje de publicaciones realizadas sin cooperación interinstitucional y las realizadas con otras instituciones ecuatorianas puede obedecer a la creciente disponibilidad de revistas científicas regionales que se encuentran en la base Latindex (Crespo-Gascón y otros, 2019), tal como se muestra en la Figura 3. Por otro lado, el alto porcentaje de publicaciones con colaboración interinstitucional se debe a que INIAP ejecuta varios proyectos con instituciones de investigación nacional e internacional, por lo cual la propiedad intelectual de la producción científica es compartida.
En orden de importancia le siguen países de las regiones de Norteamérica (6,5\%), Europa $(3,7 \%)$, ALC $(5,7 \%)$ y otras regiones $(4,9 \%)$. Además, tomando en cuenta todas las combinaciones posibles con otras regiones se logró obtener el $31,7 \%$ de los documentos publicados. Estos resultados indican una importante interacción del INIAP con otras instituciones ecuatorianas y de varias regiones para la generación de investigaciones en el campo de la agricultura.

Los principales países europeos que más colaboran con científicos del INIAP son España, Francia e Italia, con 18, 14 y 7 publicaciones bilaterales respectivamente (Tabla III). Estados Unidos y Canadá son los países norteamericanos que más colaboran con INIAP, con 44 y 5 publicaciones bilaterales respectivamente. Por otro lado, los países latinoamericanos que más colaboran con científicos del INIAP son Ecuador, México, Brasil y Colombia, con 92, 14, 10 y 8 publicaciones bilaterales respectivamente. Estos resultados corroborarían que los científicos que colaboran internacionalmente más a menudo son los Latinoamericanos, comparados con los científicos de otras partes del mundo (Nature index, 2016). De los países europeos, España es el que mayor colabora con el Instituto; España se ha convertido en un país clave para la colaboración científica internacional, sus publicaciones cientí-

Tabla II. Numero de publicaciones en colaboración entre INIAP y otros países. 2014-2019

\begin{tabular}{lcc}
\hline Tipo de cooperación & $\begin{array}{c}\text { Número de documentos } \\
\text { publicados }\end{array}$ & $\begin{array}{c}\text { Porcentaje total de } \\
\text { documentos publicados }\end{array}$ \\
\hline Solo INIAP & 39 & 15,9 \\
Solo Ecuador & 74 & 30,1 \\
Solo ALC & 14 & 5,7 \\
Solo Norteamérica & 16 & 6,5 \\
Solo Europa & 9 & 3,7 \\
Ecuador +ALC & 24 & 9,8 \\
Ecuador+Norteamérica & 21 & 8,5 \\
Ecuador+Europa & 16 & 6,5 \\
Ecuador+Norteamérica+Europa & 1 & 0,4 \\
Ecuador+ALC+Europa & 2 & 0,8 \\
ALC+Norteamérica & 6 & 2,4 \\
ALC+Europa & 6 & 2,4 \\
ALC+Norteamérica+Europa & 4 & 1,6 \\
Norteamérica+Europa & 2 & 0,8 \\
Otras regiones (Asia, África y Oceanía) & 12 & 4,9 \\
\hline Total & 246 & 100 \\
\hline
\end{tabular}


ficas internacionales han crecido, representando valores cercanos al $8 \%$ de las colaboraciones internacionales mundiales (Fundación Española para la Ciencia y Tecnología, 2016). En el caso de Norteamérica, Estados Unidos es el país que mostró el mayor número de publicaciones con participación de investigadores de INIAP, siendo la superioridad científica de Estados Unidos la razón que explica el alcance de esta colaboración.

Tomando en cuenta solo Latinoamérica, Ecuador tiene el mayor número de publicaciones en colaboración bilateral con INIAP (92 publicaciones), esto debido a la creación de redes científicas de Investigación y Desarrollo Tecnológico (I\&D) agropecuario, detalladas a continuación:

1. Red Regional de Investigación de Chocho o Tarwi, creada con el objetivo de contribuir a la articulación de la investigación científica y tecnológica regional sobre el sector de producción y consumo del chocho o tarwi; y, definir los requerimientos de investigación en el sector productivo del chocho, para la seguridad y soberanía alimentaria, considerando su alto valor nutricional, alimentario, agroindustrial y adaptabilidad a condiciones agroecológicas marginales. Cuenta con 132 miembros de 40 organizaciones y consultores privados de Ecuador, Perú, Bolivia.

2. Red de Genética y Genómica ReGG: conformada por: Universidad Yachay Tech, INIAP, INABIO, Universidad Tecnológica Equinoccial (UTE), Cruz Roja, Universidad San Francisco de Quito y la Sociedad Ecuatoriana de Genética Humana. El objetivo principal es ser un espacio para que investigadores puedan colaborar y participar de subvenciones internacionales con proyectos conjuntos

3. Plataforma Multiagencia de cacao para América Latina y el Caribe "Cacao 2030 - 2050", se crea con el objetivo de generar relaciones a largo plazo entre todos los actores de la cadena del cacao a nivel regional para constituirse en la base de nuevas iniciativas en el tiempo y a su vez integrar a los actores integrantes de este proyecto y otros actores potenciales. Los miembros son: Organización Ejecutora, Escuela Superior Politécnica del Litoral (ESPOL). Organizaciones co-ejecutoras, AGROSAVIA, Centro Agronómico Tropical de Investigación y Enseñanza (CATIE), Instituto Nacional de Innovación y Transferencia en Tecnología (INTA) de Costa Rica, Universidad Nacional de Costa Rica. Organizaciones asociadas, Instituto Nacional de Investigaciones Agropecuarias (INIAP), Instituto Nacional de Innovación
Agraria (INIA) de Perú, Centro Internacional de Agricultura Tropical (CIAT), Comité Europeo para la Formación y la Agricultura (CEFA), Ministerio de Agricultura y Ganadería de Ecuador, Deutsche Gessellschaft für Internatinale Zusammernarbeit (GIZ), Instituto Dominicano de Investigaciones Agropecuarias y Forestales (IDIAF) y Universidad Nacional de Colombia Sede Palmira.

4. Red Regional de Comunicación Agrícola, con el objetivo de reunir a investigadores, profesionales y técnicos junto a los comunicadores, quienes presentarán ejemplos de buenas prácticas comunicacionales, compartirán experiencias acerca de su trabajo de diseminación de resultados de proyectos y otras iniciativas comunicacionales. Los participantes de la red son comunicadores e investigadores de 17 países, incluido INIAP (Argentina, Bolivia, Chile, Colombia, Costa Rica, Ecuador, España, Estados Unidos, Francia, Guatemala, Honduras, Nicaragua, Panamá, Paraguay, Perú, República Dominicana, y Uruguay).

5. Plataforma de Conocimientos sobre Agricultura Familiar (PCAF), el objetivo conectar a los actores principales del mismo país o región y fomentar que estudien temas de interés común que puedan servir como base para el establecimiento de asociaciones y colaboración entre sus miembros. Adicionalmente, el INIAP como miembro tiene la oportunidad de que sus publicaciones técnicas tengan mayor visibilidad internacional a través de la Plataforma de conocimientos sobre la agricultura familiar.

El aumento del número de investigadores acreditados o registrados en el Ecuador en los últimos años ha contribuido a mejorar la colaboración interinstitucional, encontrándose en las bases de datos de la SENESCYT a nivel nacional un total de 2867 investigadores acreditados activos en el año 2019, de los cuales el 3\% corresponde al número de investigadores acreditados pertenecientes al INIAP. También, de esos 2867 investigadores acreditados, el $30 \%$ aproximadamente pertenecen al área de conocimiento de 'ciencias de la vida'. Todos estos resultados se corroboran con la normalización de los datos a través de la medida de Salton (Tabla III), con el propósito de cuantificar la importancia de la colaboración entre el INIAP y otros países incluido el Ecuador. Los resultados indicaron que el Ecuador $(0,106)$ tiene fuertes vínculos con el INIAP, seguido por México $(0,006)$, Estados Unidos $(0,005)$ y España $(0,005)$. La medida de Salton es un estimador de la fortaleza de la colaboración internacional y está definido como el número de publicaciones conjuntas entre dos países dividido 
por la raíz cuadrada del producto del número de los resultados totales de publicación de los dos países (Glänzel, 2001)

Con el fin de interpretar la importancia de la co-autoría, en la Figura 9 se muestra la afiliación de los primeros autores y autores corresponsales (por regiones) de las publicaciones científicas del INIAP. En cuanto a la afiliación del autor principal, se observó un 55\% de primeros autores pertenecientes a INIAP en las publicaciones, esto indica que más de la mitad de las publicaciones conjuntas fueron lideradas por investigadores del Instituto. Para el caso de autores corresponsales, el 50\% están afiliados a instituciones ecuatorianas y el $22 \%$ pertenecen al INIAP. El 28\% restante corresponde a instituciones de Norteamérica, Europa, Asia y América Latina y el Caribe.

\subsection{Modelo de regresión}

El análisis de regresión estadística confirmó los resultados obtenidos en este estudio. Los factores que determinaron la citación de las publicaciones científicas del INIAP se exponen en la Tabla IV.

El año de publicación determinó de manera negativa la citación de los artículos $(t=-3,15)$. La interpretación del coeficiente de esta variable indica que las publicaciones que se encuentran más tiempo en una base de datos tendrán mayor posibilidad de ser citadas, lo que corrobora lo indicado por Sánchez Pereyra y otros, (2015) quienes mencionan que el número de citas de los documentos publicados en años anteriores son más altos, una vez que los documentos son publicados inicia el periodo a partir del cual son susceptibles de ser citados y alcanzan sus niveles de citación máximos en años posteriores.

Tabla III. Número de publicaciones del INIAP con o sin colaboración, periodo 2014-2019

\begin{tabular}{lccc}
\hline Países & $\begin{array}{c}\text { Número total de } \\
\text { publicaciones }\end{array}$ & $\begin{array}{c}\text { Número de publicaciones } \\
\text { en colaboración }\end{array}$ & Medida de Salton \\
\hline Ecuador & 3073 & 92 & 0,106 \\
Estados Unidos & 295494 & 44 & 0,005 \\
España & 59162 & 18 & 0,005 \\
México & 24461 & 14 & 0,006 \\
Francia & 57452 & 14 & 0,004 \\
Brasil & 87270 & 10 & 0,002 \\
Italia & 53700 & 7 & 0,002 \\
Australia & 63858 & 7 & 0,002 \\
Colombia & 8861 & 8 & 0,005 \\
Canadá & 58447 & 5 & 0,001 \\
Dinamarca & 17048 & 5 & 0,002 \\
\hline
\end{tabular}

Figura 9. Afiliación de primeros autores y autores corresponsales de publicaciones científicas del INIAP en el periodo 2014-2018
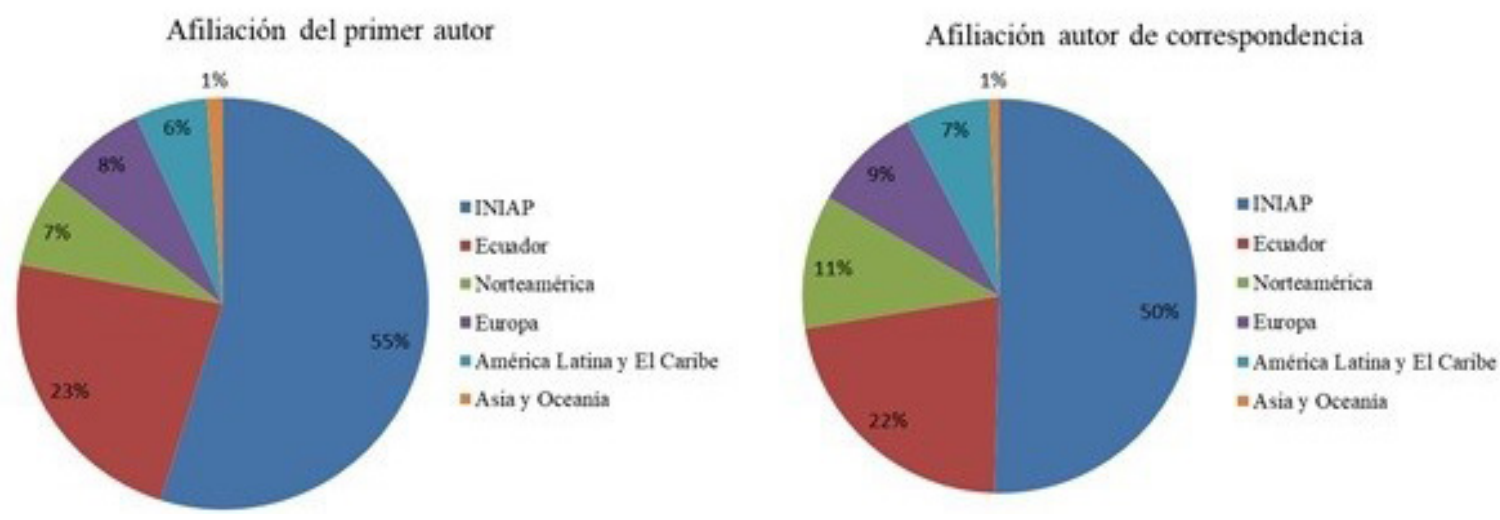
La colaboración internacional resultó importante para mejorar las posibilidades de citación ( $\mathrm{t}=$ $3,41)$, constituyendo un factor positivo para elevar el número de citas (Cobo-Sánchez y otros, 2018). Estos vínculos pueden facilitar la difusión de conocimientos y sirven como conductos a través de las cuales la información se distribuye entre la comunidad científica (Ahuja, 2000). Una publicación resultante de la mayor participación de instituciones ubicadas en distintos continentes, permite que el documento sea visualizado y mayormente citado (González-Alcaide y Gómez, 2017). La procedencia del autor (principal o de correspondencia) también influyó en la citación de los artículos. Los resultados indicaron que un autor norteamericano, tanto como principal $(t=2,16)$ así como de correspondencia ( $t$ $=2,30$ ) inciden en una mayor citación. Estos resultados denotan la relevancia de autores que provienen de sistemas de innovación en países desarrollados, en donde sus economías se basan sólidamente en ciencia, tecnología, innovación y educación avanzada (Arocena y Sutz, 2002). Además, los investigadores ecuatorianos muchas veces acuden a estas regiones del mundo para cursar sus programas de postgrado (Castillo y Powell, 2019).

Según el modelo de regresión, la publicación en "idioma inglés" es una variable que incide en la citación de un artículo científico del INIAP ( $\mathrm{t}=$ $3,53)$. El inglés es el idioma más utilizado en los trabajos científicos y es aceptado principalmente a nivel mundial para la comunicación de documentos entre países. Se estima que el $98 \%$ de las publicaciones indexadas son en este idioma y que tienen además amplia difusión en la comunidad científica (Encalada-Díaz, 2017). La visibilidad e impacto de las publicaciones científicas no solamente está garantizada por el renombre de la institución que las acoge, las empresas editoriales que dan soporte o su ubicación dentro de las distintas indexaciones y rankings internacionales, sino también por el idioma en el que fueron publicados. Además, al ser publicadas en inglés, permite ser leídas por más personas y contribuyen a mejorar la divulgación del saber científico.

Se observó que los artículos relacionados al área de investigación de "incorporación de valor agregado" tienen posibilidades de citación $(t=1,95)$. Actualmente, esta área de investigación se encuentra priorizada en el Modelo de Gestión del INIAP que tiene un enfoque de cadenas productivas, enfatizando en la agroindustria (valor agregado) (Viera y Merino, 2019); además está alineada a un plan de desarrollo impulsado por el Gobierno del Ecuador para apoyar la investigación científica a las industrias químicas, farmacéuticas y alimenticias (Instituto Nacional de Investigaciones Agropecuarias, 2014). Esta área de investigación es de interés en muchos países de Latinoamérica y El Caribe, mismos que por su tradicional condición de proveedores de materias primas buscan aumentar su participación en mercados más dinámicos y potenciales como el agroindustrial (Riveros y Heinrichs, 2014).

Un factor que a menudo resulta determinante para un alto número de citaciones es el hecho que el artículo se encuentre en una revista de acceso abierto (Cobo-Sánchez y otros, 2018). En nuestro análisis se observó que esta variable tiene un aspecto negativo para la citación $(t=-1,82)$. Este resultado se explica porque existe un gran porcentaje de documentos científicos que se encuentran en revistas indexadas que tienen un costo que debe ser cancelado por el usuario para poder acceder a ellos; sin embargo, varias instituciones tienen suscripción a las bases de datos lo que permite reducir el impacto de esta variable en la citación.

Tabla IV. Resultados de la regresión (variable dependiente expresado en $\ln +1$ ). $R^{2}=0,399$

\begin{tabular}{|c|c|c|c|c|}
\hline Variable & Coeficiente & $\begin{array}{l}\text { Error } \\
\text { estándar }\end{array}$ & Estadístico t & $p>|t|$ \\
\hline Año de publicación & $-0,17 * * *$ & 0,54 & $-3,15$ & 0,002 \\
\hline Número de continentes colaboran & $0,32 * * *$ & 0,09 & 3,41 & 0,001 \\
\hline Autor principal: Norteamérica & $0,64 * * *$ & 0,30 & 2,16 & 0,032 \\
\hline Autor correspondencia: Norteamérica & $0,56 * *$ & 0,24 & 2,30 & 0,023 \\
\hline Autor correspondencia: Europa & $0,67 * *$ & 0,28 & 2,35 & 0,020 \\
\hline Autor correspondencia: ALC & $0,27 * *$ & 0,15 & 1,70 & 0,092 \\
\hline Publicado en Inglés & $0,58^{*}$ & 0,16 & 3,53 & 0,001 \\
\hline $\begin{array}{l}\text { Área de Investigación: Incorporación de valor } \\
\text { agregado }\end{array}$ & $0,42 * *$ & 0,21 & 1,95 & 0,052 \\
\hline Revista en acceso abierto & $-0,32 *$ & 0,17 & $-1,82$ & 0,071 \\
\hline Constante & 344,71 & 109,34 & 3,15 & 0,002 \\
\hline
\end{tabular}

*** $\mathrm{p}<0,01 ; * * \mathrm{p}<0,05 ; * \mathrm{p}<0,1$ 


\section{CONCLUSIONES}

Este análisis muestra que las publicaciones científicas de INIAP en su mayoría se encuentran en revistas indexadas con índice SJR. La colaboración internacional tiene un rol relevante para la citación de los documentos generados. El área de investigación con más potencial de citación fue "incorporación de valor agregado a la producción agropecuaria", enfoque actualmente priorizado por el INIAP.

La participación de la mujer en la producción científica del Instituto ha mantenido un porcentaje estable durante el periodo analizado en este estudio, aunque se mantiene bajo comparado con el promedio en Latinoamérica, sin embargo no es el menor porcentaje en la región.

Con base en los resultados obtenidos, se recomienda el idioma inglés para la escritura de las pu-

\section{REFERENCIAS}

Abramo, G.; D'Angelo, C.A.; Solazzi, M. (2011). The relationship between scientists' research performance and the degree of internationalization of their research. Scientometrics, 86(3), 629-643. https://doi. org/10.1007/s11192-010-0284-7

Ahuja, G. (2000). Collaboration networks, structural holes, and innovation: A Longitudinal Study. Administrative Science Quarterly, 45(3), 425-455. https://doi. org/10.2307/2667105

Aleixandre-Benavent, R. (2010). Bibliometría e indicadores de actividad científica. En: J. Jiménez, M. Argimon, M. Martín y M. Vilardell (Eds). Publicación científica biomédica. Cómo escribir y publicar un artículo de investigación, pp. 363-384. Barcelona: Elsevier. https://doi.org/10.1016/b978-84-8086-4619.50027-8

Álvarez-Muñoz, P.; Pérez-Montoro, M. (2015). Análisis de la producción y la visibilidad científica de Ecuador en el contexto andino (2000-2013). El profesional de la información, 24 (5), 577-586. https://doi. org/10.3145/epi.2015.sep.07

Arocena, R.; Sutz, J. (2002). Innovation systems and devolping countries. DRUID Working Paper No. 0205. Copenhagen, Dinamarca: Copenhagen Business School, Department of Industrial Economics and Strategy/Aalborg University, Department of Business Studies.

Bindé, J. (2005). Hacia las sociedades del conocimiento: informe mundial de la UNESCO. Paris: UNESCO.

Boshoff, N. (2010). South-South research collaboration of countries in the Southern African Development Community (SADC). Scientometrics, 84 (2), 481-503. https://doi.org/10.1007/s11192-009-0120-0

Bravo-Vinaja, A.; Sanz-Casado, E. 2008. Análisis bibliométrico de la producción científica de México blicaciones para obtener un mayor impacto en la comunidad científica con el uso de la información generada.

\section{AGRADECIMIENTOS}

Los autores agradecen al Instituto Nacional de Investigaciones Agropecuarias por el apoyo y financiamiento de este estudio. Agradecemos al Dr. Lenin Ron de la Universidad Central del Ecuador por su colaboración en el análisis estadístico.

\section{ACKNOWLEDGEMENTS}

Authors thanks to the National Agricultural Research Institute for supporting and financing this study. We thank Dr. Lenin Ron from Central University of Ecuador for his support in the statistical analyses.

en ciencias agrícolas durante el periodo 1983-2002. Revista Fitotecnia Mexicana, 31 (3): 187-194.

Brunner, J; Ferrada-Hurtado, R. (2011). Educación superior en Iberoamérica. Informe 2011. Providencia, Santiago: Centro Interuniversitario de Desarrollo (Cinda).

Cañedo-Andalia, R. (1999). Los análisis de citas en la evaluación de los trabajos científicos y las publicaciones seriadas. ACIMED, 7(1), 30-39. Disponible en: https:// bit.ly/330ezIG.

Castillo, J.; Powell, M. (2019). Análisis de la producción científica del Ecuador e impacto de la colaboración internacional en el periodo 2006-2015. Revista Española de Documentación Científica, 42 (1), e225. https://doi.org/10.3989/redc.2019.1.1567

Catanzaro, M.; Guiliano Miranda, L.; Alezu B. (2014). South America science: Big players. Nature, 510 (7504), 204-206. https://doi.org/10.1038/510204a

Chaple, A. (2015). Fotografía clínica estomatológica: consejos para la práctica diaria. Revista Cubana de Estomatología., 52, 80-83.

Cobo Sánchez, J.; Ochando García, A.; Blanco Mavillard, I.; Cirera Segura, F.; Crespo Montero, R.; Casas Cuesta, R. (2018). Análisis del impacto de la producción científica de la revista Enfermería Nefrológica entre 1998 y 2017. Enfermería Nefrológica, 21 (4), 349-358.

Coccia, M.; Rolfo, S. (2007). How research policy changes can affect organization and productivity of public research institutes? Analysis within the Italian national system of innovation. Journal of Comparative Policy Analysis: Research and Practice, 9 (3), 215-233. https://doi. org/10.1080/13876980701494624 
Crespo-Gascón, S.; Tortosa, F.; Guerrero-Casado, J. (2019). Producción de revistas científicas en América Latina y El Caribe en Scopus, Journal Citation Reports y Latindex en el área de los recursos naturales: su relación con variables económicas, ambientales y de inversión en investigación. Revista Española de Documentación Científica, 42 (1), e224. https://doi. org/10.3989/redc.2019.1.1533

De Granda-Orive, J. I.; Alonso-Arroyo, A.; García-Río, F.; Solano-Reina, S.; Jiménez-Ruiz, C. A.; AleixandreBenavent, R. (2013), Ciertas ventajas de Scopus sobre Web of Science en un análisis bibliométrico sobre tabaquismo. Revista Española de Documentación Científica, 36 (2): e011, doi: https://doi.org/10.3989/ redc.2013.2.941

Encalada-Díaz, M. (2017). ¿Por qué escribir y por qué en inglés?. Acta ortopédica mexicana, 31(3), 107. Disponible en: https://bit.ly/2IjGIrS

Fundación Española para la Ciencia y la Tecnología (2016). Indicadores bibliométricos de la actividad científica española 2005-2014. pp:13-14. España: FECYT.

García y Pérez, E. (2017). Las "mentiras" científicas sobre las mujeres. Madrid: Los libros de la Catarata.

Glänzel, W. (2001). National characteristics in international scientific co-authorship relations. Scientometrics, 51 (1), 69-115. https://doi. org/10.1023/A: 1010512628145

González-Albo, B.; Aparicio, J.; Moreno, L.; Bordons, M. (2016). Los sectores institucionales en la producción científica española de difusión internacional. Revista Española de Documentación Científica, 39(1), enero-marzo 2016, e115. https://doi.org/10.3989/ redc. 2016.1 .1260

Gonzalez-Alcaide, G; Gómez, J. (2017). Análisis de las prácticas de colaboración científica: una vía hacia la excelencia. Valencia: Nau Llibres.

Hassan, S-U.; Sarwar, R.; Muazzam, A. (2015). Tapping into intra- and international collaborations of the Organization of Islamic Cooperation states across science and technology disciplines. Science and Public Policy, 43 (5), 690-701. https://doi.org/10.1093/ scipol/scv072

Inglesi-Lotz, R.; Pouris, A. (2011). Scientometric impact assessment of a research policy instrument: the case of rating researchers on scientific outputs in South Africa. Scientometrics, 88, 747-760. https://doi. org/10.1007/s11192-011-0440-8

Instituto Nacional de Investigaciones Agropecuarias (INIAP). (2014). Plan estratégico de investigación, desarrollo e innovación 2014-2017. Quito: Instituto Nacional de Investigaciones Agropecuarias.

Kreimer, P.; Heimer V. (2018). Latin American science, technology, and society: A historical and reflexive approach. Tapuya: Latin American Science, Technology and Society, 1 (1), 17-37. https://doi.org/10.1080/25 729861.2017 .1368622
Markovsky, I.; Van Huffel, S. (2007). Overview of total least-squares methods. Signal Processing, 87(10), 2283-2302. https://doi.org/10.1016/j. sigpro.2007.04.004

Marques de Sá, J. (2007). Applied Statistics Using SPSS, STATISTICA, MATLAB and $R$ ( $2^{\mathrm{a}}$ ed.). Heidelberg: Springer. https://doi.org/10.1007/9783-540-71972-4

Medina, J.; Cordero L.; Carrillo P.; Rodriguez, D.; Castillo, J. A.; Astudillo, I.; Cárdenas, S.; Trinidad, E. de; Powell, M. (2016). Investigación científica. En: Ramírez R. (ed.). Universidad urgente para una sociedad emancipada, pp 461-494. Quito, Ecuador: SENESCYT-IESALC.

Muller, K. y Fetterman, B. (2002). Regression and ANOVA: An integrated approach using SAS software. North Carolina: SAS Institute Inc.

Naranjo, M. (2017). INIAP- La investigación agropecuaria: Trascendencia, implicaciones y desafíos (Evaluación impactos INIAP). Quito: Instituto Nacional de Investigaciones Agropecuarias.

Nature index. (2016). Disponible en: http://www. natureindex.com [Fecha de consulta: 27/09/2019].

Organización de las Naciones Unidas para la Educación, la Ciencia y la Cultura (UNESCO). (2015). UNESCO science report: Towards 2030. Paris, Francia: United Nations Educational, Scientific and Cultural Organization.

Pessina, M. (2019). Ecuador: Investigadoras en ciencia y tecnología atrapadas entre la invisibilización y el androcentrismo. En: Impacto de las mujeres en la ciencia efecto del género en el desarrollo y la práctica científica, pp: 29-55. Quito, Ecuador: Ciespal. ISBN digital: 978-9942-8777-0-3.

Pouris, A.; Ho, Y.S. (2014). Research emphasis and collaboration in Africa. Scientometrics, 98 (3), 21692184. https://doi.org/10.1007/s11192-013-1156-8

Pu, Q. H.; Lyu, Q. J.; Su, H. Y. (2016). Bibliometric analysis of scientific publications in transplantation journals from Mainland China, Japan, South Korea and Taiwan between 2006 and 2015. BMJ Open, 6, e011623. https://doi.org/10.1136/bmjopen-2016-011623

Quinde-Rosales, V.; Bucaram, R.; Bucaram, M.; Silvera, C. 2019. Relación entre el gasto en Ciencia y Tecnología y el Producto Interno Bruto. Un análisis empírico entre América Latina y el Cribe y el Ecuador. Revista Espacios $10(4), 7$.

Red de Indicadores de Ciencia y Tecnología Iberoamericana e Interamericana (RICYT). (2018). El Estado de la Ciencia. Principales indicadores de ciencia y tecnología Iberoamericanos / Interamericanos. Buenos Aires, Argentina: UNESCO, REDES.

Ríos, C.; Herrero, V. (2005). La producción científica latinoamericana y la ciencia mundial: una revisión bibliográfica (1989-2003). Revista Interamericana de Bibliotecología, 28 (1), 43-61. 
Riveros, H.; Heinrichs, W. (2014). Valor agregado en los productos de origen agropecuario: aspectos conceptuales y operativos. San José, C.R.: IICA.

Sánchez, V.; Zambrano, J. (2019). Adopción e impacto de las tecnologías agropecuarias generadas en el Ecuador. La Granja: Revista de Ciencias de la Vida 30 (2), 2839. https://doi.org/10.17163/Igr.n30.2019.03

Sánchez Pereyra, A.; Carrillo Romero, O; Garrido Villegas, P. (2015). Análisis bibliométrico de la Revista Mexicana de Sociología basado en indicadores de citación. Revista Mexicana de Sociología 77: 45-70.

Secretaría de Educación Superior, Ciencia, Tecnología e innovación (SENESCYT) e Instituto Nacional de Estadísticas y Censos (INEC). (2014). Indicadores de Actividades de Ciencia, Tecnología e Innovación (ACTI) del Ecuador, Período 2009-2014. Quito, Editogram Medios Públicos.

Secretaría de Educación Superior, Ciencia, Tecnología e innovación. 2018. Lineamientos para establecer el nivel de las publicaciones indexadas para el proceso de categorización y recategorización de investigadores. Quito, Ecuador.

Thelwall, M. (2018). Using Almetrics to Support Research Evaluation. En: Erdt M.; et al. (Eds.), AROSIM 2018, CCIS 856, pp. 11-28. Singapore: Springer. https:// doi.org/10.1007/978-981-13-1053-9_2
Tinoco, K. (2018). El INIAP y su importancia en el sector agrícola. Quito:INIAP. ISBN: 978-9942-22-338-8.

Viera, W.; Merino, J. (2019). Direccionamiento del modelo de gestión I+D+i INIAP 2019: Enfoque cadena de valor y sistemas de producción. Reporte Técnico. Quito: Instituto Nacional de Investigaciones Agropecuarias.

Yanez, F.; Estrella, D.; Robalino, G.; Carrillo, R. (2019). Plan estratégico institucional 2019-2022. Quito: INIAP. ISBN: $978-9942-22-45-2$.

Zambrano, J.; Barrera, B.; Murillo, I.; Domínguez, J. (2018). Plan estratégico de investigación y desarrollo tecnológico del INIAP 2018-2022. Quito: INIAP.

Zambrano, J. (2019). La sociedad del conocimiento: cantidad, categoría y género de los investigadores en Ecuador. Mundos Plurales: Revista Latinoamericana de Políticas y Acción Pública, 6 (1). https://doi. org/10.17141/mundosplurales.1.2019.3855

Zubieta, J. (2007). Las científicas latinoamericanas y sus avatares para posicionarse en la esfera de la ciencia y la tecnología. En: Sebastián, J. (ed.). Claves del desarrollo científico y tecnológico en América Latina. Madrid: Siglo XXI. 